\title{
Notas sobre a nidificação da galinha-d'água, Gallinula galeata (Lichtenstein, 1818) (Gruiformes, Rallidae)
}

Notes on the nesting habits of the Common Gallinule, Gallinula galeata (Lichtenstein, 1818) (Gruiformes, Rallidae)

\author{
Maria Eliana Carvalho Navega-Gonçalves (1) \\ Grupo de Trabalho Interdisciplinar sobre Fauna Silvestre. Secretaria de Defesa do Meio Ambiente. Piracicaba, São Paulo, Brasil
}

\begin{abstract}
Resumo: A galinha-d'água, Gallinula galeata, é uma ave de área úmida, comumente encontrada em quase todo o Brasil. Entre março e abril de 2019, observei e descrevi dois ninhos construídos pela espécie no Parque da Rua do Porto, em Piracicaba, estado de São Paulo. O primeiro ninho foi construído sobre a água, próximo à vegetação da margem do lago e parcialmente ocultado por ela, enquanto o segundo, construído sobre um banco de areia no lago, se encontrava exposto. Ambos eram constituídos de materiais semelhantes, com predomínio de ramos vegetais de espessuras variadas, além de cascas de árvore, gramíneas, musgos, raízes, folhas e frutos. Material plástico foi encontrado nos dois ninhos. Selecionei 60 imagens de ninhos da espécie disponíveis no site WikiAves - uma plataforma online brasileira para compartilhamento de registros ornitológicos - para comparação com aqueles que observei. As análises mostraram variedade de materiais utilizados na elaboração dos ninhos, bem como diferentes locais de nidificação, tanto em ambiente aquático como terrestre, sugerindo plasticidade fenotípica. $O$ manejo adequado do parque, visando à conservação dos sítios de nidificação e à manutenção dos recursos, é essencial para a sobrevivência desta e de outras aves em ambientes antropizados.
\end{abstract}

Palavras-chave: Avifauna. Ninho. Material antropogênico. Sítio de nidificação.

\begin{abstract}
The Common Gallinule, Gallinula galeata, is a bird from humid areas, commonly found in almost entire Brazil. Between March and April 2019 I observed and described two nests built by the species in the Rua do Porto Park, in the city of Piracicaba, São Paulo state. The first nest was built on the water, near lakeshore vegetation and partially hidden by it, while the second, built on a sandbank in the lake, was exposed. Both were made of similar materials, with a predominance of vegetable branches of varying thickness, as well as tree bark, grasses, mosses, roots, leaves and fruits. Plastic material was found in both. I selected 60 images of nests of the species available on the WikiAves website - a Brazilian online platform for sharing ornithological records- for comparison with the ones that I observed. The analyses showed a variety of materials used in the nest building, as well as different nesting sites, both in aquatic and terrestrial environments, suggesting phenotypic plasticity. The proper park management, which aims at the conservation of nesting sites and resources, is essential to the survival of this and other birds in anthropized environments.
\end{abstract}

Keywords: Avifauna. Nest. Anthropogenic material. Nesting site.

NAVEGA-GONÇALVES, M. E. C., 2021. Notas sobre a nidificação da galinha-d'água, Gallinula galeata (Lichtenstein, 1818) (Gruiformes, Rallidae). Boletim do Museu Paraense Emílio Goeldi. Ciências Naturais 16(1): 145-155. DOI: http://doi.org/10.46357/bcnaturais.v16i1.442. Autora para correspondência: Maria Eliana Carvalho Navega-Gonçalves. Rua Manoel da Silva Garcia, 59 - Campestre. Piracicaba, SP, Brasil. CEP 13401-724 (eliana.navega@gmail.com).

Recebido em 13/10/2020

Aprovado em 02/03/2021

Responsabilidade editorial: Adriano Oliveira Maciel
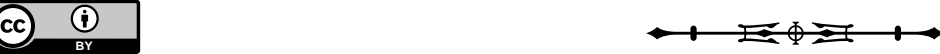


\section{INTRODUÇÃO}

A nidificação é a atividade de construção de ninhos (do latim: nidificare, que significa 'fazer ninho'), sendo um mecanismo complexo e fundamental para o ciclo de vida das aves, por estar ligado à reprodução destes animais (Altamirano et al., 2012). A confecção do ninho pelas aves tem como objetivo a postura, a incubação e o cuidado e a proteção de ovos e ninhegos (Hansell, 2000; Altamirano et al., 2012). Portanto, o material utilizado é organizado para adequação dos ovos e da ave que irá chocá-los, além da acomodação dos filhotes enquanto estiverem no ninho (Silveira, 2012).

Há uma grande variedade de materiais usados na construção dos ninhos, sendo principalmente de origem vegetal, alguns de origem animal e uma minoria apresentando material inorgânico e de origem antrópica (Hansell, 2000). Assim, a seleção de sítios de nidificação e a oferta de materiais necessários para a construção de ninhos são fatores que influenciam no sucesso reprodutivo das aves (Li \& Martin, 1991; Filliater et al., 1994; Deeming \& Mainwaring, 2015; Healy et al., 2015) e, como consequência, a permanência destas em determinado ambiente e localidade.

Este estudo teve como objetivo descrever alguns aspectos do comportamento de nidificação de Gallinula galeata (Lichtenstein, 1818) (antes Gallinula chloropus Linnaeus, 1758) em um parque urbano em Piracicaba, São Paulo, Brasil, e comparar os ninhos observados com ninhos fotografados e publicados no site de ciência cidadã Wikiaves (2020). A galinha-d'água ou frango-d'água-comum, como também é conhecida, é uma espécie cosmopolita e comum em todo o Brasil, com exceção da Amazônia, onde é mais difícil de ser encontrada; habita coleções de água com farta vegetação paludícola e arbustiva na borda (Höfling \& Camargo, 2002; Sigrist, 2014), sendo, portanto, uma ave tipicamente de área úmida (Accordi, 2010). Possui hábito alimentar onívoro (Beltzer et al., 1989) e, geralmente, nidifica sobre vegetação aquática flutuante ou nas margens das coleções d'água (Sigrist, 2014), podendo construir o ninho em terra a poucos metros de distância da água (Brito, 2012).

\section{MATERIAL E MÉTODOS}

As observações sobre a nidificação de Gallinula galeata foram realizadas de maneira esporádica no Parque da Rua do Porto "João Herrmann Neto", entre os meses de março e abril de 2019, como parte de um projeto de levantamento e monitoramento das aves do parque encaminhado à Secretaria Municipal de Defesa do Meio Ambiente (SEDEMA), Piracicaba, São Paulo.

O parque situa-se no bairro Chácara Nazareth ( $22^{\circ} 43^{\prime} 36,9^{\prime \prime}$ S, 47 39' 38,2” W), na área urbana do município de Piracicaba, São Paulo (Figura 1), e possui

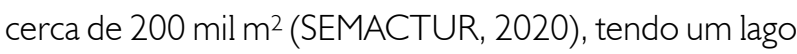
central com aproximadamente $1.250 \mathrm{~m}$ de perímetro e 30 mil $\mathrm{m}^{2}$ de área, medidos pelo software Google Earth (s. d.).

As margens do lago são delimitadas por vegetação rasteira (gramíneas) e arbustiva, em alguns trechos, sendo parcialmente sombreadas pelas árvores do entorno, características que favorecem a manutenção de uma pequena população da espécie do estudo (Figura 2). O lago está sujeito a assoreamento, o que provoca a formação de bancos de areia e, muito embora esta situação possa comprometer a fauna aquática, para algumas aves os bancos de areia servem de locais estratégicos para obtenção de alimento e para nidificação (Navega-Gonçalves \& Trevisan, 2020).

Com o intuito de comparar a estrutura dos ninhos de Gallinula galeata observados no parque com aqueles construídos em outros locais, analisei imagens de ninhos da espécie, oriundos de áreas úmidas disponibilizadas no site WikiAves, e selecionei 60 imagens, cujos ninhos continham ovos ou ninhegos e/ou adultos, nos quais os materiais constituintes estavam visíveis. Estas referem-se a imagens obtidas apenas nos sete estados do Sul e Sudeste do Brasil, uma vez que os registros de ninhos para outras

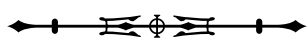


regiões são poucos no site. Para fazer a comparação, estabeleci três critérios: 1) material predominante visualizado na estrutura do ninho (descrito na seção de
Resultados e Discussão); 2) se o ninho se encontrava 'exposto', 'semiexposto' ou 'ocultado'; e 3) o local onde o ninho havia sido construído (Tabela 1).

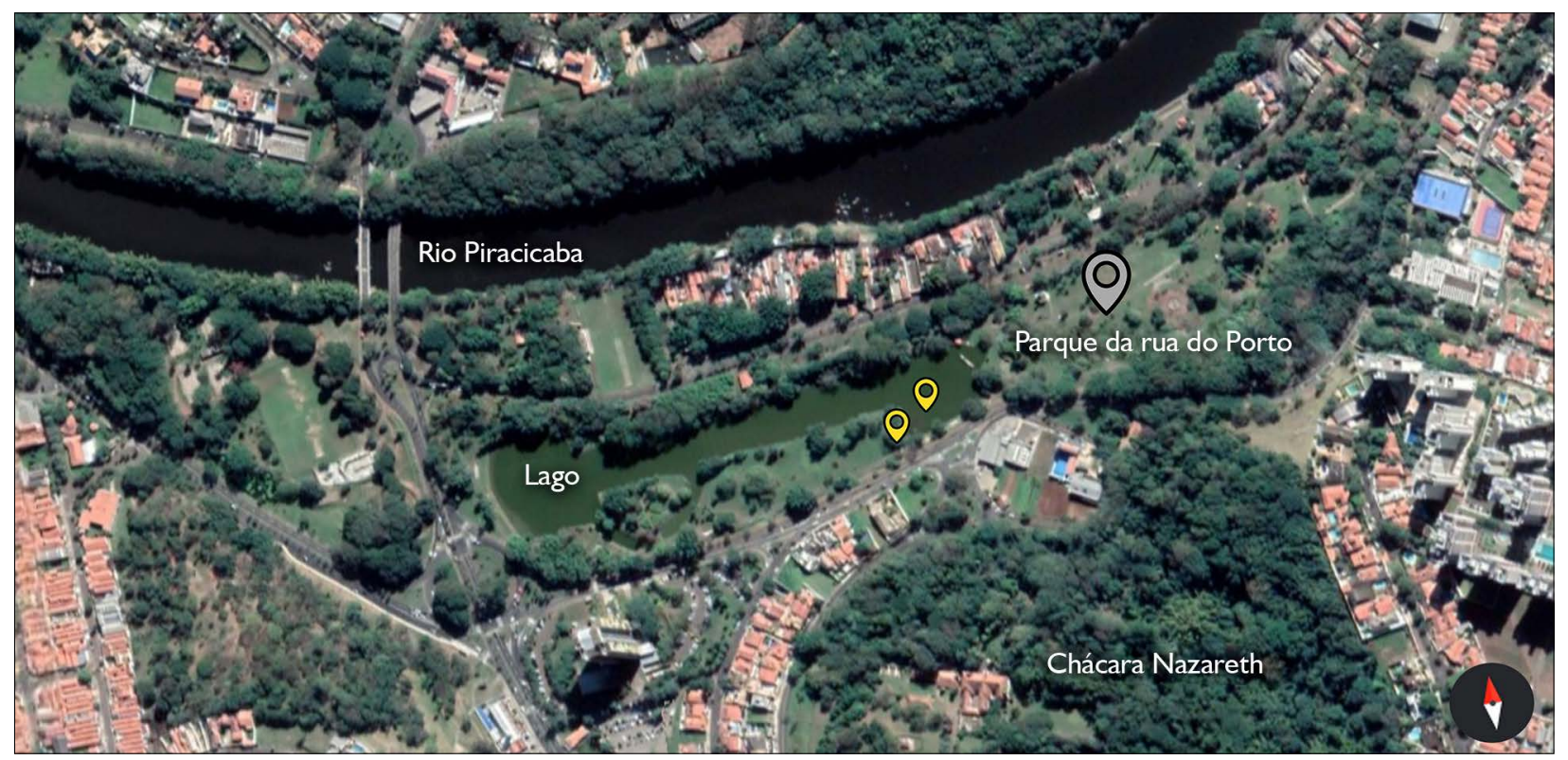

Figura 1. Parque da Rua do Porto, Piracicaba, São Paulo. Os marcadores amarelos indicam os locais onde foram observados os ninhos. Fonte: modificada de Google Earth (s. d.).

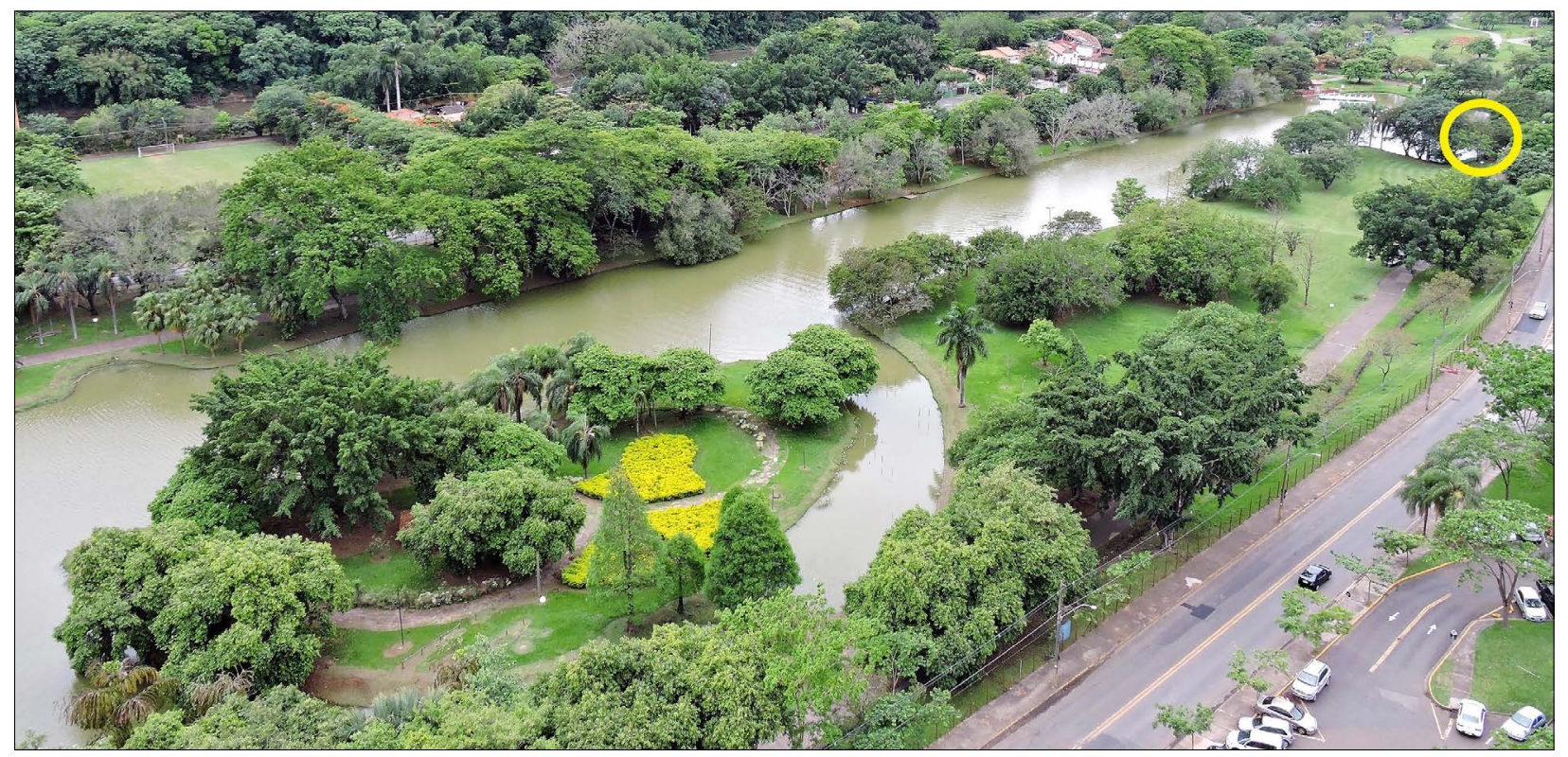

Figura 2. Vista aérea do Parque da Rua do Porto, Piracicaba, São Paulo, mostrando o lago e a vegetação do entorno. $\bigcirc$ círculo amarelo indica a área onde os ninhos de Gallinula galeata foram localizados. Foto: Maria Eliana C. Navega-Gonçalves (2018).

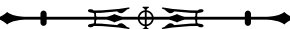


Tabela 1. Dados relativos às imagens de ninhos de Gallinula galeata selecionadas da galeria de fotos da espécie no site WikiAves. Legendas: Local - sobre a água, vegetação aquática ou tronco na água (Aq.); sobre a vegetação terrestre ou sobre o solo (Te.); exposto (Ex.); semiexposto (Se.); oculto (Oc.); material antropogênico visível (Ma.); * = o ninho foi classificado como terrestre por estar sobre uma estrutura de cimento.

\begin{tabular}{|c|c|c|c|c|c|c|c|c|}
\hline Imagem & Município/Estado & Ano & Aq. & Te. & Ex. & Se. & Oc. & Ma. \\
\hline WA1473752 & Marechal Floriano/ES & 2011 & & $x$ & $x$ & & & \\
\hline WA514689 & Formosa do Sul/SC & 2011 & $x$ & & $x$ & & & \\
\hline WA1546409 & Vila Velha/ES & 2014 & & $x$ & $x$ & & & \\
\hline WA3571988 & Guarulhos/SP & 2019 & $x$ & & $x$ & & & \\
\hline WA3265189 & Sorocaba/SP & 2019 & $x$ & & $x$ & & & \\
\hline WA2715604 & Resplendor/MG & 2016 & $x$ & & $x$ & & & \\
\hline WA451644 & Curitiba/PR & 2011 & & $x$ & & & $x$ & \\
\hline WA1697319 & São Paulo/SP & 2015 & $x$ & & $x$ & & & \\
\hline WA1125718 & Barueri/SP & 2013 & & $x$ & $x$ & & & $x$ \\
\hline WA111450 & São Paulo/SP & 2010 & $x$ & & $x$ & & & \\
\hline WA3756181 & Cotia/SP & 2019 & & $x$ & $x$ & & & \\
\hline WA1153337 & São Paulo/SP & 2013 & & $x$ & & $x$ & & \\
\hline WA1882764 & Iracemápolis/SP & 2015 & & $x$ & $x$ & & & \\
\hline WA1665296 & São Paulo/SP & 2015 & $x$ & & $x$ & & & \\
\hline WA1248116 & Cambará do Sul/RS & 2014 & $x$ & & $x$ & & & \\
\hline WA1181150 & Porto União/SC & 2013 & $x$ & & $x$ & & & \\
\hline WA301017 & Rio Claro/SP & 2002 & & $x$ & & $x$ & & \\
\hline WA23763 & Americana/SP & 2009 & $x$ & & & $x$ & & \\
\hline WA3179750 & Laguna/SC & 2018 & & $x$ & $x$ & & & \\
\hline WA1246754 & Florianópolis/SC & 2014 & & $x$ & & $x$ & & \\
\hline WA1140861 & Curitiba/SP & 2013 & $x$ & & $x$ & & & \\
\hline WA1079868 & Belo Horizonte/MG & 2013 & $x$ & & $x$ & & & \\
\hline WA805486 & Curitiba/PR & 2012 & $x$ & & & $x$ & & \\
\hline WA246118 & Curitiba/PR & 2010 & $x$ & & $x$ & & & \\
\hline WA5958 & Tapiraí/SP & 2009 & $x$ & & $x$ & & & \\
\hline WA1294 & Curitiba/PR & 2003 & & $x$ & $x$ & & & \\
\hline WA3471799 & Ipatinga/MG & 2019 & $x$ & & $x$ & & & \\
\hline WA1501726 & Espírito Santo do Pinhal/SP & 2014 & $x$ & & $x$ & & & \\
\hline WA817231 & Derrubadas/RS & 2012 & $x$ & & & $x$ & & \\
\hline WA693482 & Rio Grande/RS & 1997 & & $x$ & & $x$ & & \\
\hline WA501010 & Barueri/SP & 2011 & $x$ & & $x$ & & & \\
\hline WA3198393 & Júlio de Castilhos/RS & 2018 & & $x$ & $x$ & & & \\
\hline WA3434684 & Jundiaí/SP & 2019 & $x$ & & $x$ & & & \\
\hline WA3269010 & Gaspar/SC & 2019 & & $x$ & $x$ & & & \\
\hline WA3209911 & Santa Bárbara D'Oeste/SP & 2018 & & $x$ & & $x$ & & \\
\hline
\end{tabular}


Tabela 1.

(Conclusão)

\begin{tabular}{|c|c|c|c|c|c|c|c|c|}
\hline Imagem & Município/Estado & Ano & Aq. & Te. & Ex. & Se. & Oc. & Ma. \\
\hline WA2473020 & Vila Valério/ES & 2017 & & $x$ & $x$ & & & \\
\hline WA2126275 & Domingos Martins/ES & 2016 & $x$ & & $x$ & & & \\
\hline WA1862848 & São Paulo/SP & 2015 & $x$ & & $x$ & & & \\
\hline WA1680459 & São Paulo/SP & 2015 & $x$ & & $x$ & & & \\
\hline WA1494071 & Florianópolis/SC & 2014 & $x$ & & $x$ & & & \\
\hline WA1389252 & Vitória/ES & 2014 & & $x$ & & $x$ & & \\
\hline WA1160751 & Itapuí/SP & 2013 & & $x$ & & $x$ & & \\
\hline WA3632827 & Curitiba/PR & 1999 & $x$ & & $x$ & & & \\
\hline WA484729 & São Miguel D'Oeste/SC & 2011 & $x$ & & & $x$ & & \\
\hline WA229759 & Canela/RS & 2010 & $x$ & & $x$ & & & \\
\hline WA84131 & São Paulo/SP & 2009 & $x$ & & & $x$ & & \\
\hline WA3675243 & Derrubadas/RS & 2020 & $x$ & & $x$ & & & \\
\hline WA3632828 & Curitiba/PR & 2020 & $x$ & & $x$ & & & \\
\hline WA3546989 & Jaraguá do Sul/SC & 2019 & $x$ & & $x$ & & & \\
\hline WA3600296 & Juiz de Fora/RJ & 2019 & $x$ & & $x$ & & & \\
\hline WA3545502 & Caraguatatuba/SP & 2019 & $x$ & & & $x$ & & \\
\hline WA3519163 & Tubarão/SC & 2019 & $x$ & & $x$ & & & \\
\hline WA1501471 & Salto/SP & 2014 & $x$ & & $x$ & & & \\
\hline WA3255154 & Laurentino/SC & 2019 & $x$ & & $x$ & & & \\
\hline WA2720228* & Belo Horizonte/MG & 2017 & & $x$ & & $x$ & & $x$ \\
\hline WA2483538 & São Bento do Sul/RS & 2011 & & $x$ & $x$ & & & \\
\hline WA2456922 & Campinas/SP & 2017 & $x$ & & $x$ & & & \\
\hline WA2003529 & São João D'Oeste/SC & 2016 & & $x$ & & $x$ & & \\
\hline WA1160751 & Itapuí/SP & 2013 & & $x$ & & $x$ & & \\
\hline WA758241 & São Paulo/SP & 2010 & $x$ & & & $x$ & & \\
\hline Total: 60 & - & - & 38 & 22 & 42 & 17 & 1 & 2 \\
\hline
\end{tabular}

\section{RESULTADOS E DISCUSSÃO}

Observei dois ninhos de galinha-d'água no Parque da Rua do Porto em 2019. O primeiro ninho, observado no dia 2 de março, estava sendo construído sobre a água e junto à vegetação da margem sul do lago. No momento da observação, a ave deslocava-se sobre o lago buscando ramos vegetais (galhos), que transportava no bico e depositava sobre uma base recém-construída de matéria vegetal. O local escolhido apresentava vegetação alta ocultando parte do ninho.
Um adulto chegou a ser observado sobre o ninho no mesmo dia (Figura 3).

No dia 20 de março, este ninho foi encontrado abandonado, com sua estrutura visivelmente desarranjada e foi, então, coletado. Não havia vestígios de ovos e a análise mostrou que era constituído por ramos vegetais de tamanhos e espessuras diversas, cascas de árvore, gramíneas, musgos, raízes, folhas e frutos (do tipo vagem), além de material plástico identificado como sendo uma sacola plástica rasgada e de cor amarela (Figura 4). 


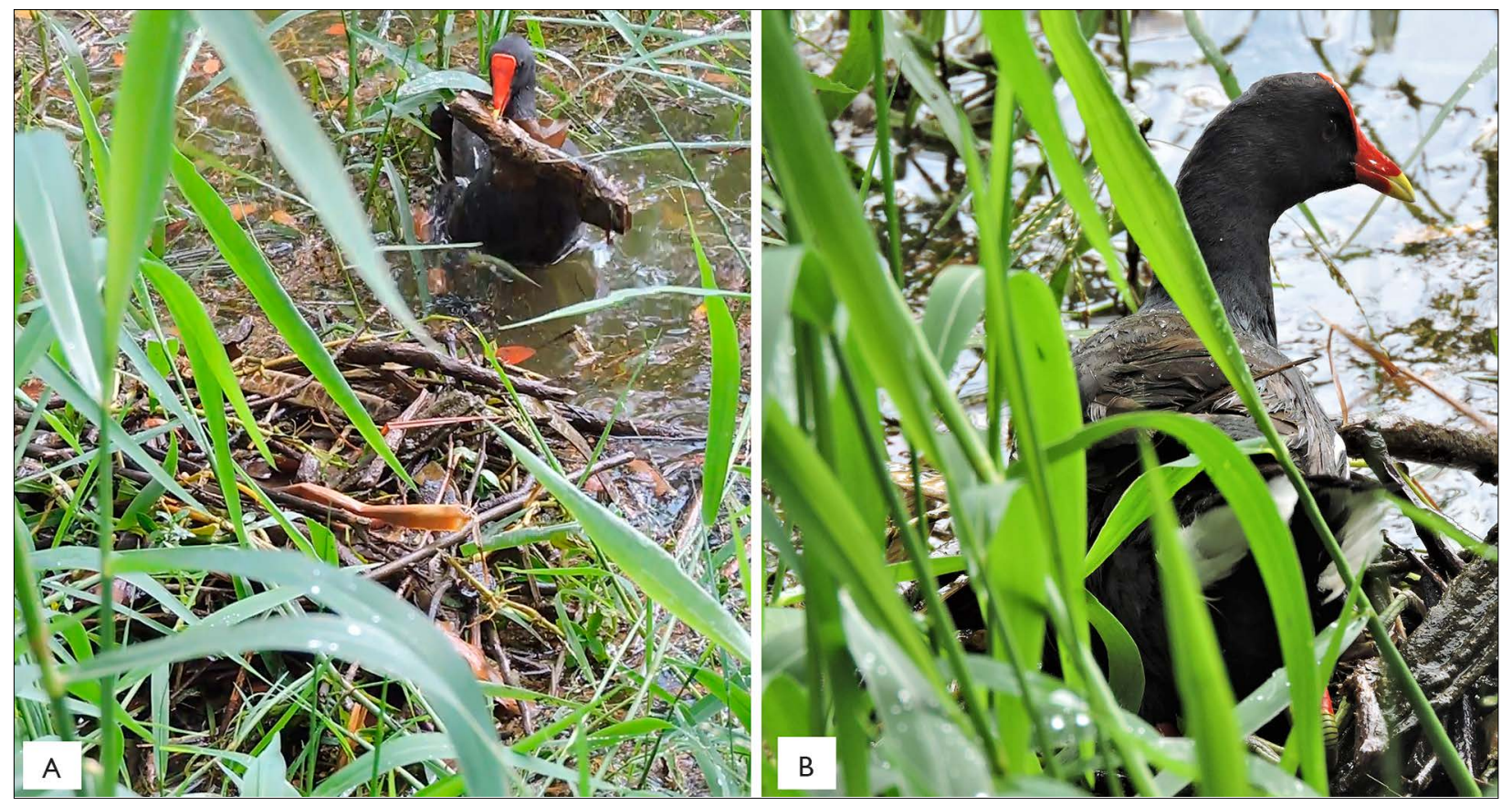

Figura 3. A) Indivíduo de Gallinula galeata transportando galhos para o ninho em construção na margem do lago do Parque da Rua do Porto, Piracicaba, São Paulo, Brasil; B) adulto sobre o ninho recém-construído. Fotos: Maria Eliana C. Navega-Gonçalves (2019).

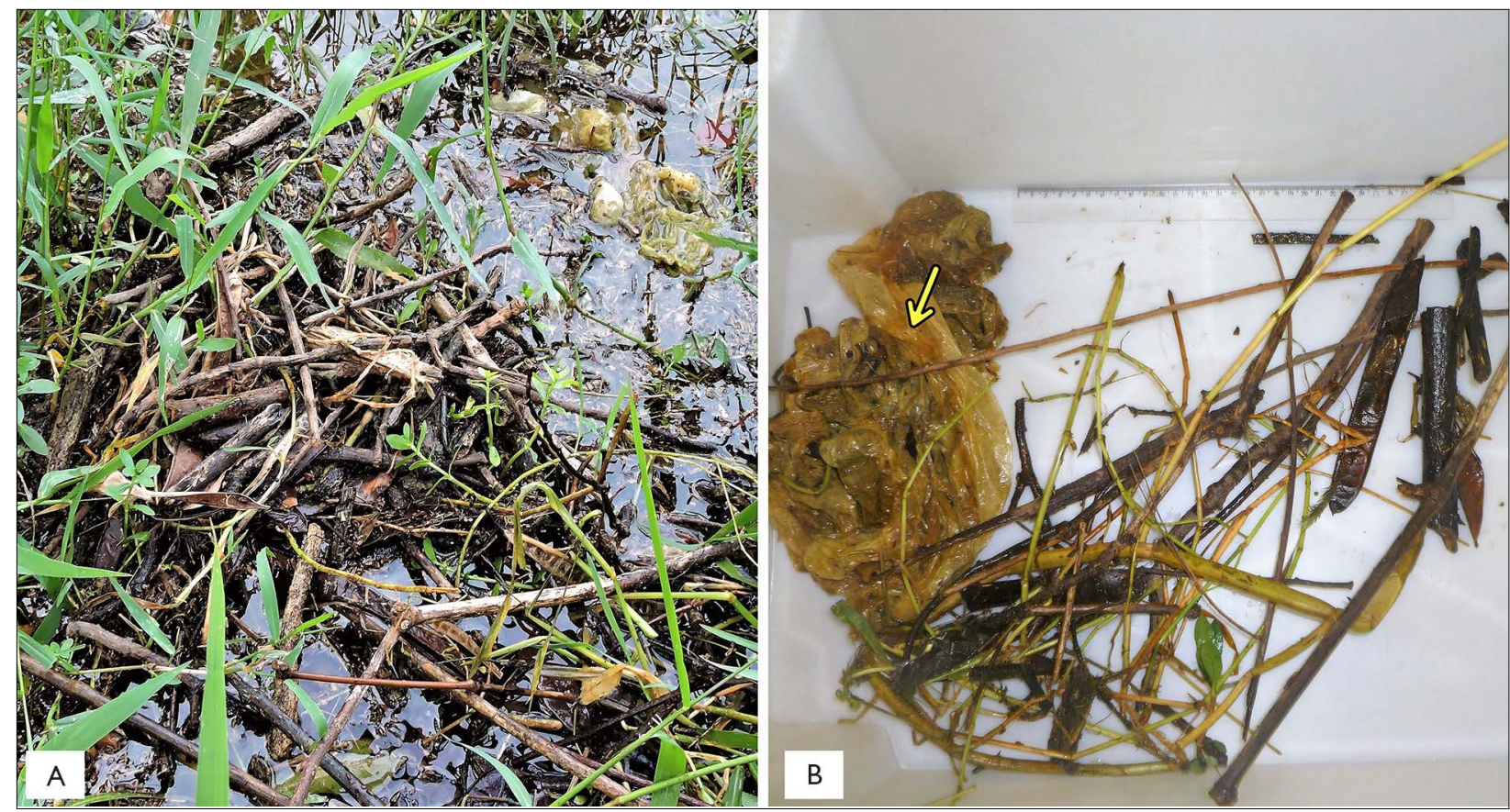

Figura 4. A) Ninho de Gallinula galeata abandonado e com sua estrutura desarranjada; B) parte do ninho coletado para análise dos materiais constituintes: a seta indica o material plástico. Fotos: Maria Eliana C. Navega-Gonçalves (2019) (A); Letícia Steagall Zanatta (2019) (B).

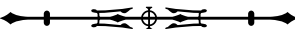


Avistei o segundo ninho sobre um banco de areia, a cerca de 9 metros da margem sul do lago, no dia 29 de março. Este encontrava-se totalmente exposto, uma vez que no local não havia vegetação. Era formado, principalmente, por ramos vegetais de tamanhos e espessuras distintas, sendo que os ramos da base eram bem maiores e mais espessos do que aqueles da parte superior do ninho. Também compunham a estrutura do ninho gramíneas ainda vivas, folhas secas, frutos (do tipo vagem) e material plástico não identificado. No mesmo dia, observei dois adultos próximos ao ninho e, em seguida, um deles incubando os ovos (que não foram visualizados). Posteriormente, no dia 7 de abril, avistei um filhote no ninho junto a um dos adultos, quando a água já encobria quase todo o banco de areia, alcançando as bordas do ninho (Figura 5). No dia 10 de abril, o ninho já havia sido abandonado e sua estrutura estava praticamente desfeita.

Não foi possível determinar se o indivíduo que construiu o primeiro ninho, às margens do lago, era o mesmo que construiu o ninho no banco de areia, após o insucesso do primeiro. Não foi possível nem mesmo dizer se os adultos avistados junto aos ninhos eram o macho, a fêmea ou ambos, uma vez que não há dimorfismo sexual nesta espécie. Segundo Helm et al. (1987), os ninhos das galinhas d'água são construídos por ambos os sexos e o monoformismo sexual pode estar relacionado ao fato de que ambos os pais participam na criação e no cuidado da prole (Fenoglio et al., 2002). Entre as imagens do WikiAves, algumas mostram dois adultos, geralmente um deles sobre o ninho e o outro próximo ao mesmo, evidenciando a participação do casal no cuidado da prole (e.g. WA1079868).

Os dois ninhos foram construídos com materiais semelhantes, com predomínio de galhos de espessuras variadas. No entanto, o primeiro ninho, que foi armado diretamente sobre a água, tinha em sua base um entrelaçado de caules ainda vivos de plantas encontradas na própria margem do lago (gramíneas) (Figura 4B), sobre o qual os demais materiais foram sendo depositados, enquanto o segundo, elaborado sobre o banco de areia,
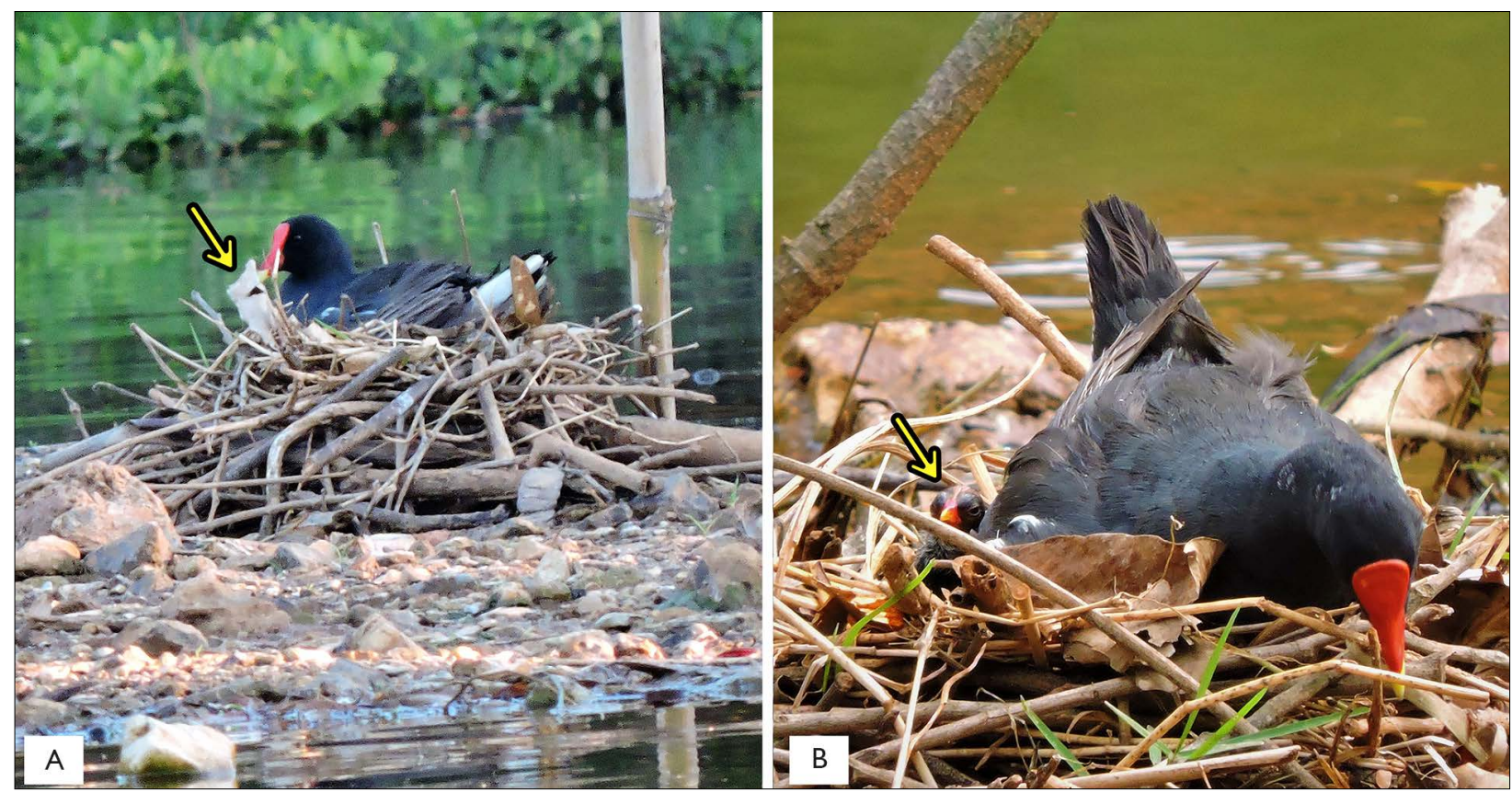

Figura 5. Ninho de Gallinula galeata construído sobre um banco de areia no lago: A) adulto chocando os ovos (a seta indica material plástico); B) adulto com filhote (indicado pela seta) no ninho. Fotos: Maria Eliana C. Navega-Gonçalves (2019).

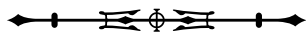


continha basicamente galhos secos arranjados uns sobre os outros (Figura 5A).

Analisando as imagens dos ninhos de Gallinula galeata obtidas no WikiAves (Tabela 1), foi possível constatar que há grande variedade de materiais utilizados em sua elaboração, com predomínio de galhos de espessuras diversas e gramíneas (secas ou vivas) em sua estrutura básica. Também identifiquei, nas imagens, plantas aquáticas vivas, folhas vivas e secas, além de frutos do tipo vagem em alguns dos ninhos. Em duas imagens, foi possível detectar a presença de material antropogênico no ninho, fitas plásticas em ambos os casos, além de um canudo em um deles (WA1125718 e WA2720228).

Nas imagens analisadas, 38 ninhos estavam na água, sobre a vegetação aquática ou sobre tronco na água, e 22 sobre a vegetação terrestre ou sobre o solo (Tabela 1). Os ninhos construídos sobre a água apresentam uma estrutura mais volumosa quando comparados àqueles construídos fora da água, provavelmente devido à maior quantidade de material depositado, de forma a constituir uma sólida e alta plataforma flutuante, que pode estar diretamente sobre a água ou apoiada sobre plantas aquáticas ou plantas encontradas nas margens dos cursos d'água. Os ninhos construídos fora da água e sobre a vegetação rasteira (gramínea) são formados basicamente pela própria planta, na maioria das vezes, seca (palha), como forração, sem outro tipo de material agregado, exceto algumas folhas de árvores/arbustos, observadas em alguns. Os ninhos construídos sobre o solo tinham como base, predominantemente, galhos e folhas secos. Os ninhos terrestres são rasos e visivelmente menos elaborados.

Estas diferenças observadas entre os ninhos construídos em substratos diferentes dificultam a sua classificação nas categorias existentes. Segundo a padronização proposta por Simon \& Pacheco (2005), os ninhos de Gallinula galeata inserem-se no tipo 'cesto baixo/base', cujos ninhos assemelham-se a uma cesta ou tigela, com altura menor ou igual ao diâmetro externo. No entanto, alguns ninhos avistados sobre o solo ou a vegetação terrestre são tão simples que poderiam ser classificados como sendo do tipo 'simples/plataforma', no qual os materiais usados na construção estão frouxamente entrelaçados para formar uma base que amortece os ovos sobre o substrato (Simon \& Pacheco, 2005).

Alguns estudos mostram o predomínio de ninhos construídos pelas galinhas-d'água sobre ou entre a vegetação aquática (Helm et al., 1987; Forman \& Brain, 2004; Brito, 2012; Samraoui et al., 2013). No entanto, nestes trabalhos, há também relatos de ninhos construídos sobre a vegetação terrestre e, ocasionalmente, em arbustos e árvores próximos às áreas úmidas. Forman \& Brain (2004) destacam que, onde a vegetação é frequentemente manejada, estas aves também nidificam em árvores, muitas vezes a 2,5 m do nível do solo. Entre as imagens analisadas no WikiAves, não havia registro de ninho construído sobre arbustos ou árvores no ambiente terrestre.

A maioria dos ninhos observados a partir das imagens encontrava-se exposta $(n=42)$, estando aqueles construídos sobre a água bem visíveis. Parte daqueles que se encontravam sobre a vegetação terrestre ou próximos às margens dos cursos d'água estava semiexposta $(n=17)$. Apenas um ninho estava bem ocultado pela vegetação do entorno (Tabela 1).

A presença da vegetação próxima ao ninho dificulta o reconhecimento do mesmo por parte de um predador. Forman \& Brain (2004) constataram que a altura da vegetação acima dos ninhos de Gallinula chloropus influencia positivamente no sucesso da reprodução. Além disso, o tipo de material usado na construção do ninho, sua estrutura básica, o tamanho e a sua localização podem minimizar a predação (Bailey et al., 2015; Mainwaring et al., 2015).

Dentro do parque, o número de predadores é menor do que em um ambiente natural, no entanto, existem ali predadores de ovos, como o teiú (Tupinambis Daudin 1802) (Mazzotti et al., 2014), e de ovos/filhotes, como o falcão Caracara plancus (Miller, 1777) e os gaviões Buteo brachyurus Vieillot, 1816 e Rupornis magnirostris (Gmelin, 1788) (Sick, 1997; Sigrist, 2014), para os quais 
os ninhos expostos facilitam a predação. A presença do teiú foi registrada através de observação pessoal, e as aves citadas foram identificadas no levantamento da avifauna do parque (Navega-Gonçalves \& Trevisan, 2020). Na imagem WA3756181 do WikiAves (Tabela 1), foi registrado um teiú predando os ovos da espécie em estudo.

Há evidências de que a escolha do material para a construção dos ninhos, por várias espécies de aves, seja oportunista e que as mesmas se utilizem, principalmente, de material de origem vegetal disponível nas proximidades (Helm et al., 1987; Surgey et al., 2012; Briggs \& Deeming, 2016). É possível, ainda, que variações encontradas entre os ninhos de uma mesma espécie se deem em decorrência do local onde o mesmo foi construído, sugerindo plasticidade fenotípica, conforme apontam os estudos citados.

uso de material antropogênico tem sido relatado em ninhos de várias espécies de aves, sendo que o plástico vem mostrando uma prevalência maior em relação a outros materiais (Roda \& Carlos, 2003; Vasconcelos et al., 2006; Cristofoli \& Sander, 2007; Cristofoli et al., 2008; Tomaz et al., 2009; Costa \& Mäder, 2011; Pereira, 2011; Scherer et al., 2012; Chaves et al., 2013; Townsend \& Barker, 2014; Batisteli et al., 2019), provavelmente pela frequência com que é utilizado na confecção de materiais diversos pelo homem e pelo descarte inapropriado no ambiente. A poluição causada pelo plástico é considerada uma das maiores ameaças à fauna (Jagiello et al., 2019) e, no caso das aves, este tipo de material pode evidenciar a presença do ninho chamando a atenção de predadores (Canal et al., 2016), além de ser um risco para os filhotes, que podem ingerir pequenas partículas ou se emaranhar em sacos, fitas e linhas de pesca (Townsend \& Barker, 2014).

Embora haja vários estudos sobre o comportamento reprodutivo das aves abordando os diversos padrões de construção de ninhos, seleção de materiais e de sítios de nidificação (Hansell, 2000; Deeming \& Mainwaring, 2015; Healy et al., 2015; Biddle et al., 2018a, 2018b), algumas questões sobre o tema permanecem sem respostas.
Alguns estudos foram realizados com Gallinula chloropus/ G. galeata (Helm et al., 1987; Post \& Seals, 2000; TelinoJúnior et al., 2003; Forman \& Brain, 2004; Wallau et al., 2010; Samraoui et al., 2013; Rodríguez-Casanova \& Zuria, 2018), no entanto, aspectos do comportamento de nidificação necessitam ser elucidados, sugerindo futuras investigações, com o intuito de responder às seguintes questões: 1) $\bigcirc$ que induziria alguns indivíduos da espécie a construírem o ninho sobre a água, outros sobre a vegetação terrestre ou diretamente sobre o solo?; 2) É possível estabelecer uma relação entre a disponibilidade de recursos e a estrutura do ninho, bem como a escolha do local para a nidificação?; 3) Outros fatores poderiam estar envolvidos nesta escolha, tais como proteção contra predação e/ou disponibilidade de alimento para os filhotes? Segundo Biddle et al. (2018b), estudos detalhados sobre os tipos de materiais usados na construção de ninhos pelas aves limitam-se a algumas espécies e aqueles que analisam as propriedades estruturais dos mesmos são raros. Assim, é plausível que a coleta e a análise de ninhos inativos se constituam como uma excelente oportunidade para ampliar o conhecimento sobre o assunto.

O Parque da Rua do Porto, conforme apontado por Navega-Gonçalves e Trevisan (2020), é uma área importante para a avifauna, que encontra ali refúgio e/ou habitat, em meio a uma matriz urbana. $\bigcirc$ manejo adequado do parque, visando à conservação dos sítios de nidificação e à manutenção de seus recursos, é essencial para a sobrevivência desta e de outras aves, uma vez que o sucesso reprodutivo delas depende dos recursos disponíveis no local e da proteção que o mesmo oferece aos ovos e aos filhotes.

\section{AGRADECIMENTOS}

Agradeço aos dois revisores anônimos, pelas valiosas contribuições; à Secretaria Municipal de Defesa do Meio Ambiente (SEDEMA) de Piracicaba, São Paulo, pelo apoio às pesquisas realizadas no parque; e à Camila Navega Gonçalves, pela edição das imagens. 


\section{REFERÊNCIAS}

ACCORDI, I. A., 2010. Pesquisa e conservação de aves em áreas úmidas. In: S. V. MATTER, F. C. STRAUBE, I. A. ACCORDI, V. Q. PIACENTINI \& J. F. CÂNDIDO JR. (Org.): Ornitologia e conservação: ciência aplicada, técnicas de pesquisa e levantamento: 191-216. Technical Books, Rio de Janeiro.

ALTAMIRANO, T. A., J. T. IBARRA, F. HERNÁNDEZ, I. ROJAS, J. LAKER \& C. BONACIC, 2012. Hábitos de nidificación de las aves del bosque templado andino de Chile: 1-113. Fondo de Protección Ambiental, Ministerio del Medio Ambiente, Pontificia Universidad Católica de Chile, Santiago.

BAILEY, I. E., F. MUTH, K. MORGAN, S. L. MEDDLE \& S. D. HEALY, 2015. Birds build camouflaged nests. The Auk 132(1): 11-15. DOI: https://doi.org/10.1642/AUK-14-77.1

BATISTELI, A. F., R. GUILHERMO-FERREIRA \& H. SARMENTO, 2019. Abundance and prevalence of plastic twine in nests of Neotropical farmland birds. The Wilson Journal of Ornithology 131(1): 201-205. DOI: https://doi.org/10.1676/18-24

BELTZER, A. H., R. A. SABATTINI \& M. C. MARTA, 1989. Ecologia alimentaria de la polla de agua negra Gallinula chloropus galeata (Aves: Rallidae) en un ambiente lenitico del Rio Parana Medio, Argentina. Ornitologia Neotropical 2(1): 29-36.

BIDDLE, L. E., R. E. BROUGHTON, A. M. GOODMAN \& D. C. DEEMING, 2018a. Composition of bird nests is a species-specific characteristic. Avian Biology Research 11(2): 132-153. DOI: https:// doi.org/10.3184/175815618X15222318755467

BIDDLE, L. E., D. C. DEEMING \& A. M. GOODMAN, 2018b. Birds use structural properties when selecting materials for different parts of their nests. Journal of Ornithology 159: 999-1008. DOI: https:// doi.org/10.1007/s10336-018-1571-y

BRIGGS, K. B. \& D.C. DEEMING, 2016. Use of materials in nest construction by pied flycatchers Ficedula hypoleuca reflects localized habitat and geographical location. Bird Study 63(4): 516-524. DOI: https://doi.org/10.1080/00063657.2016.1238867

BRITO,M.R., 2012.Contributoparaoestudoeconservaçãodaespécie galinha-d'água Gallinula chloropus (Linnaeus, 1758): 1-97. Dissertação (Mestrado em Gestão e Conservação da Natureza) - Universidade dos Açores, Angra do Heroísmo. Disponível em: https://repositorio. uac.pt/bitstream/10400.3/2001/1/DissertMestradoMarianaReis Brito2013.pdf. Acesso em: 18 setembro 2019.

CANAL, D., M. MULERO-PÁZMÁNY, J. J. NEGRO \& S. FABRIZIO, 2016. Decoration increases the conspicuousness of raptor nests. PLoS ONE 11(7): e0157440. DOI: https://doi.org/10.1371/journal. pone. 0157440

CHAVES, A. V., R. G. ALVIM \& R. A. G. LOPES, 2013. O uso de materiais industriais em ninhos de Certhiaxis cinnamomeus (Passeriformes: Furnariidae) na cidade de Cláudio, Minas Gerais, Brasil. Atualidades Ornitológicas (173): 6-8.
COSTA, A. S. \&A. MÄDER, 2011. Utilização de metal na construção de ninho do sabiá-do-campo (Mimus saturninus Lichtenstein, 1823). Biodiversidade Pampeana 9(1): 1-2.

CRISTOFOLI, S. I. \& M. SANDER, 2007. Composição do ninho de corruíra: Troglodytes musculus Naumann, 1823 (Passeriformes: Troglodytidae). Biodiversidade Pampeana 5(2): 6-8.

CRISTOFOLI, S. I., C. R. SANTOS, S. A. GARCIA \& M. SANDER, 2008. Composição do ninho de cambacica: Coereba flaveola Linnaeus, 1758 (Aves: Emberezidae). Biodiversidade Pampeana 6(1): 30-33.

DEEMING, D. C. \& M. C. MAINWARING, 2015. Functional properties of nests. In: D. C. DEEMING \& S. J. REYNOLDS (Ed.): Nest, eggs, and incubation: new ideas avian reproduction: 29-49. Oxford University Press, Oxford, UK.

FENOGLIO, S., M. CUCCO \& G. MALACARNE, 2002. Bill colour and body condition in the Moorhen Gallinula chloropus. Bird Study 49(1): 89-92. DOI: https://doi.org/10.1080/00063650209461249

FILLIATER, T. S., R. BREITWISCH \& E. P. M. NEALEN, 1994. Predation on northern-cardinal nests. Does choice of nest-site matter? The Condor 96(3): 761-768.

FORMAN, D. W. \& P. F. BRAIN, 2004. Reproductive strategies used by moorhens (Gallinula chloropus) colonizing an artificial wetland habitat in south Wales. Journal of Natural History 38(3): 389-401. DOI: https://doi.org/10.1080/0022293021000010341

GOOGLE EARTH, [s. d.]. Parque da Rua do Porto - Piracicaba - SP. Disponível em: https://earth.google.com/web/. Acesso em: 04 outubro 2020.

HANSELL, M., 2000. Bird nests and construction behaviour: 1-280. Cambridge University Press, New York.

HEALY, S. D., K. V. MORGAN \& I. E. BAILEY, 2015. Nest construction behaviour. In: D. C. DEEMING \& S. J. REYNOLDS (Ed.): Nest, eggs, and incubation: new ideas avian reproduction: 16-28. Oxford University Press, Oxford, UK.

HELM, R. N., D. N. PASHLEY \& P. J. ZWANK, 1987. Notes on the nesting of the common moorhen and purple gallinule in Southwestern Louisiana. Journal of Field Ornithology 58(1): 55-61.

HÖFLING, E. \& H. F. A. CAMARGO, 2002. Aves no campus da Cidade Universitária Armando de Salles Oliveira: 3. ed.: 1-157. Editora da Universidade de São Paulo, São Paulo.

JAGIELLO, Z., L. DYLEWSKI, M. TOBOLKA \&J. I. AGUIRRE, 2019. Life in a polluted world: A global review of anthropogenic materials in bird nests. Environmental Pollution 251: 717-722. DOI: https:// doi.org/10.1016/j.envpol.2019.05.028

LI, P. \& T. E. MARTIN, 1991. Nest-site selection and nesting success of cavitynesting birds in high elevation forest drainages. The Auk 108(2): 405-418.

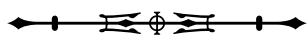


MAINWARING, M. C., S. J. REYNOLDS \& K. WEIDINGER, 2015. The influence of predation on the location and design of nests. In: D. C. DEEMING \& S.J. REYNOLDS (Ed.): Nest, eggs, and incubation: new ideas avian reproduction: 50-64. Oxford University Press, Oxford, UK.

MAZZOTTI, F. J., M. MCEACHERN, M. ROCHFORD, R. N. REED, J. K. ECKLES, J. VINCI, J. EDWARDS \& J. WASILEWSKI, 2014. Tupinambis merianae as nest predators of crocodilians and turtles in Florida, USA. Biological Invasions 17: 47-50. DOI: https://doi. org/10.1007/s10530-014-0730-1

NAVEGA-GONÇALVES, M. E. C. \& L. C. TREVISAN, 2020. Avifauna do Parque da Rua do Porto, Piracicaba, São Paulo, Brasil. Bioterra 21(2): 33-51. Disponível em: https://pt.slideshare.net/ revistabioterra/artigo-bioterra-v21n205. Acesso em: 3 agosto 2020.

PEREIRA, G. A., 2011. Ninhos de Certhiaxis cinnamomeus (Passeriformes, Furnariidae) construídos com material metálico em Pernambuco, Brasil. Atualidades Ornitologicas (159): 4-6.

POST, W. \& C. A. SEALS, 2000. Breeding biology of the common moorhen in an impounded cattail marsh. Journal of Field Ornithology 71(3): 437-442. DOI: https://doi.org/10.1648/02738570-71.3.437

RODA, S. A. \& C. J. CARLOS, 2003. On a nest of Yellow-chinned Spinetail (Certhiaxis cinnamomea - Passeriformes, Furnariidae) built with bites of wire. Lundiana 4(1): 69-70.

RODRÍGUEZ-CASANOVA, A. J. \& I. ZURIA, 2018. Aspectos reproductivos de la gallineta frente roja (Gallinula galeata) y la gallareta americana (Fulica americana) en un humedal urbano del centro de México. In: A. RAMÍREZ-BAUTISTA \& R. PINEDA-LÓPEZ (Ed.): Ecología y conservación de fauna en ambientes antropizados: 5-19. REFAMA-CONACYT-UAQ, Querétaro, México.

SAMRAOUI, F., A. H. ALFARHAN \& B. SAMRAOUI, 2013. Status and breeding ecology of the Common Moorhen Gallinula chloropus in Algeria. Journal of African Ornithology 84(2): 137-144. DOI: https://doi.org/10.2989/00306525.2013.823130

SCHERER, A. L., J. F. M. SCHERER \& M.V. PETRY, 2012. Distribuição e estrutura trófica de aves em gradiente industrial petroquímico, no Sul do Brasil. Neotropical Biology and Conservation 7(3): 162-170. DOI: http://dx.doi.org/10.4013/nbc.2012.73.03

SECRETARIA MUNICIPAL DA AÇÃO CULTURAL E TURISMO DE PIRACICABA (SEMACTUR), 2020. Prefeitura do município de Piracicaba. Parque da Rua do Porto. Disponível em: http://semactur. piracicaba.sp.gov.br/ponto_turistico/parque-da-rua-do-porto-joaohermann-neto/. Acesso: $0 \overline{7}$ julho 2020.
SICK, H., 1997. Ornitologia brasileira: 1-862. Editora Nova Fronteira, Rio de Janeiro.

SIGRIST, T., 2014. Avifauna brasileira: guia de campo Avis Brasilis: 4. ed.: 1-608. Avis Brasilis, Vinhedo, SP.

SILVEIRA, L. F., 2012. Mundo das aves: tempo de ninhos! Cães e Cia 400: 48-49. Disponível em: https://www.caes-e-cia.com.br/loja/ edicao-400-1223.html. Acesso em: 30 março 2019.

SIMON, J. E. \& S. PACHECO, 2005. On the standardization of nest descriptions of neotropical birds. Revista Brasileira de Ornitologia 13(2): 143-154.

SURGEY, J., C. R. DU FEU \& D. C. DEEMING, 2012. Opportunistic use of a wool-like artificial material as lining of tit (Paridae) nests. The Condor 114(2): 385-392. DOI: https://doi.org/10.1525/ cond.2012.110111

TELINO-JÚNIOR, W. R., S. M. AZEVEDO-JÚNIOR \& R. M. L. NEVES, 2003. Biologia e censo de Porphyrula martinica, Gallinula chloropus e Jacana jacana em Dois Irmãos, Pernambuco, Brasil. Lundiana 4(1): 43-49.

TOMAZ, V. C., V. M. FERNANDES \& M. A. S. ALVES, 2009. Reprodução de Fluvicola nengeta (Tyrannidae) em área urbana da cidade do Rio de Janeiro, RJ, Brasil. Revista Brasileira de Ornitologia 17: 70-72.

TOWNSEND, A. K. \& C. M. BARKER, 2014. Plastic and nest entanglement of urban and agricultural crows. PLoS ONE 9(1): e88006. DOI: https://doi.org/10.1371/journal.pone.0088006

VASCONCELOS, M. F., M. S. WERNECK \& M. R. DE LA PEÑA, 2006. Observações sobre a construção de um ninho de cavalaria (Paroaria capitata) com material industrializado. Revista Brasileira de Ornitologia 14(2): 167-168.

WALLAU, G. L., F. DELLA-FLORA, A. S. BUENO, J. CORSO, M. F. ORTIZ \& N. C. CÁCERES, 2010. Behaviour of the Common Moorhen in Rio Grande do Sul, Brazil. Acta Ethologica 13(2): $127-$ 135. DOI: https://doi.org/10.1007/s10211-010-0082-5

WIKIAVES, 2020. A enciclopédia das aves do Brasil: frangod'água-comum. Disponível em: https://www.wikiaves.com.br/wiki/ frango-d_agua-comum. Acesso em: 24 setembro 2020. 
